\title{
An New Automatic Connection and Separaton Device for Modularized and Fast Combined Interstage
}

\author{
Zhou Bei, Hui Junpeng, Zeng Liang \\ China Academy of Launch Vehicle Technology, Beijing, China \\ Email address: \\ 29878758@qq.com (Zhou Bei), hjpbuaa@126.com (Hui Junpeng)
}

To cite this article:

Zhou Bei, Hui Junpeng, Zeng Liang. An New Automatic Connection and Separaton Device for Modularized and Fast Combined Interstage. Science Discovery. Vol. 6, No. 5, 2018, pp. 344-352. doi: 10.11648/j.sd.20180605.16

Received: July 16, 2018; Accepted: September 18, 2018; Published: October 18, 2018

\begin{abstract}
The assembling time of traditional spacecraft's main connecting sections is long and separation structure of interstage can only be used once, which can't meet the needs for modularization ,fast combination ,little impact force and reuse. This thesis proposed a new automatic connection and sepearation device, which not only meet the demands above but also integrate the connection device and separation device. Software Pro/engineer is used to design the device's structure and software Ansys is used to analyze the strength. The results show that the structure schreme is reasonable ,the device can realize fast combination,little impact force and reuse, and the device can meet the requirements of load and small radial room.
\end{abstract}

Keywords: Spacecraft, Cabin, Connection, Seperation, Automatic

\section{适用于级间段模块化快速组合的新型舱段自动对接与分离装置}

周蓓, 惠俊鹏, 曾亮

中国运载火箭技术研究院, 北京, 中国

\section{邮箱}

29878758@qq.com（周蓓）, hjpbuaa@126.com（惠俊鹏）, z14208@163.com（曾亮）

摘要：传统航天器部段连接结构装配时间长, 且级间分离结构一次性使用, 不能满足目前航天器级间段模块化快速组 合要求以及分离冲击小可重复使用要求。因此本文提出了一种新型的舱段快速对接与分离装置, 不仅可以满足使用需 求还可将舱段快速对接结构与级间分离结构一体化。文中利用 $\mathrm{PRO} / \mathrm{E}$ 软件对该装置进行了结构设计, 并利用有限元软 件ANSYS进行了强度分析, 结果表明: 该装置结构方案设计合理, 机构在电机驱动下可快速将舱段对接并可重复分离 且冲击力小, 且可满足载荷以及狭小径向空间设计需求。

关键词: 航天器, 舱段, 对接, 分离, 自动

\section{1. 引言}

通常航天器的部段连接结构和级间分离结构分别是 航天器的不同结构。航天器的部段连接结构用于连接相邻 两个舱段, 目前舱段之间通常采用套接或轴向对接, 然后 用螺钉紧固。部段连接结构以手工装配为主, 装配效率较
低、装配周期长，不能满足快速作战级间段模块化组合的 要求。航天器的级间分离结构是将航天器的各级连接成一 个整体, 在需要分离时需要解锁, 然后在分离力的作用下 级间分离。传统航天器级间分离装置大都采用火工装置作 为驱动器, 但火工装置存在安全性差, 工作时产生较大的 冲击等问题, 且只能一次性使用, 不能重复测试性能级间 
分离结构。本文提供了一种舱段自动对接分离机构, 将对 接结构与分离结构一体化设计, 满足级间段模块化快速组 合、分离冲击小以及可重复使用等要求。

\section{2. 对接/分离装置结构及原理}

如图1所示, 为确保航天器的上下两舱段快速套装, 在两舱段之间设置雉形接触面。在上舱段的雉形面上设置 两个轴向对称的限位沟槽, 在下舱段的雉形面上相应设置 两个轴向对称的凸台。上 舱段和下舱段通过该两个轴向 对称的限位结构实现相位对准和周向定位。如图 2 所示。



图1 舱段的套接与对中。

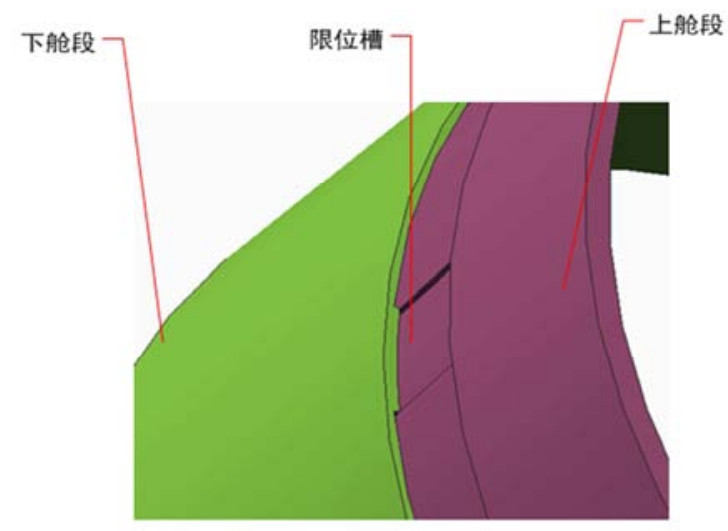

图2 舱段周向定位。

快速连接和分离机构固定安装在下舱段, 在上舱段底 部设置有环形凸台，作为锁紧连接的承压面。多个相同的 连接与分离机构沿舱段圆周方向均匀分布, 以实现对承压 面的均匀、稳定锁固, 为了便于介绍, 本方案中只展示了 其中一套。

快速连接与分离机构的结构组成如图3所示。其中, 压紧手抓、固定支架、导轨、连杆、滑块和电机均安装在 下舱段上。固定支架、导轨与电机固定安装在下舱段, 压 紧手抓、连杆、滑块为活动部件。压紧手爪与固定支架通 过销轴连接, 压紧手抓结构如图4所示, 固定支架结构如 图5所示。压紧手抓末端通过销轴与连杆连接, 连杆的另
一端通过销轴与滑块连接。滑块与导轨之间为移动副。电 机伸缩杆推动滑块运动, 并通过连杆, 实现压紧手爪对上 舱段承压面的压紧和释放。整个机构输入运动为电机伸缩 杆的直线伸缩运动, 输出运动为压紧手抓的摆动。

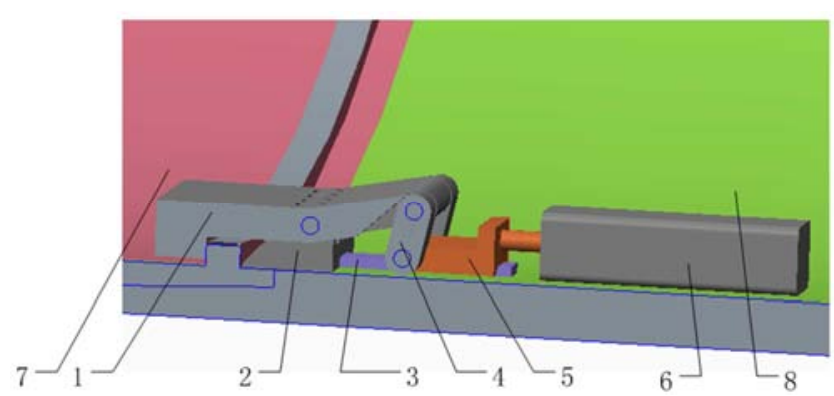

图3 舱段快速连接和分离机构结构示意图。

1、压紧手抓 2、固定支架 3、导轨4、连杆 5、滑块 6、电机 7 、上舱 段 8、下舱段

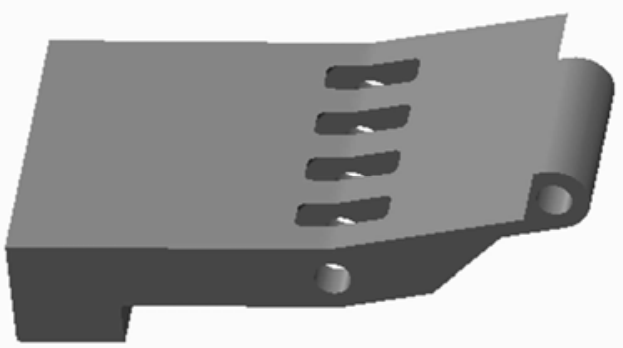

图4 抓紧手抓结构图。

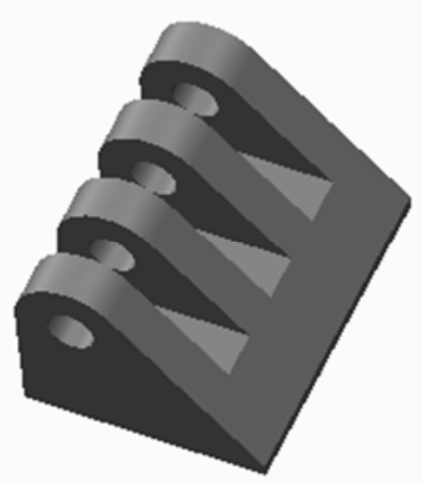

图5 固定支架结构图。

舱段快速连接和分离机构结构压紧零点状态如图6所 示。当控制系统发出舱段对接的信号，电机杆向右直线运 动。在电机杆 $\mathrm{F}$ 力的作用下, 滑块迅速向右滑动, 随之连 杆带动压紧手抓向上舱段环形凸台压去, 如图7所示。压 紧手抓接触压紧面, 此时连杆与滑块的夹角 $\theta$ 接近 $90^{\circ}$ 。在 $\theta$ 接近 $90^{\circ}$ 时, 压紧手抓接触压紧面但无预紧力。而当电机 杆向右运动到 $\theta=90^{\circ}$ 时, 压紧手抓压紧压紧面, 施加预紧 力 $\mathrm{F}_{\mathrm{y}}$, 此时电机杆不受力, 如图8所示。因此, 该机构可 有效保证上舱段与下舱段的连接与紧固。当航天器在飞行 过程或者垂直吊装过程中, 工作载荷 $\mathrm{Q}$ 主要由压紧手抓、 支架以及连杆承担, 电机杆不受力, 如图9所示。当上舱 
段与下舱段需要分离时, 电机杆向左伸出, 电机只需提供 一个极小的推动力。上下舱段对接紧固以及分离过程中以 及在各种工作状态下, 电机的驱动力 $\mathrm{F}$ 只需克服各铰链中
滚动轴承的摩擦阻力矩, 机构工作所需的驱动力 $\mathrm{F}$ 很小, 可由微型电机提供。

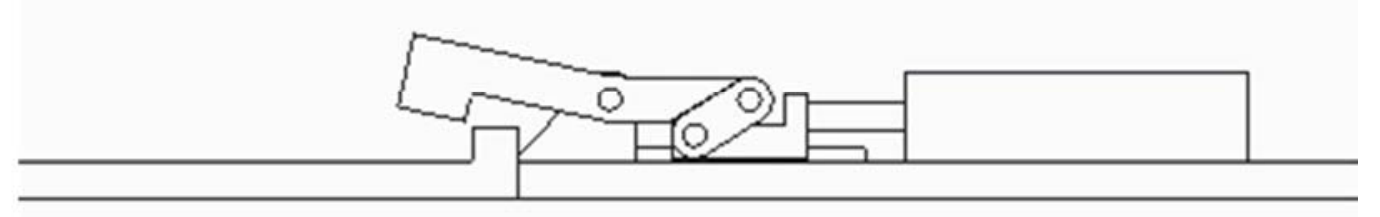

图6 舱段快速连接和分离机构结构起始状态。



图7 舱段快速连接过程机构运动。

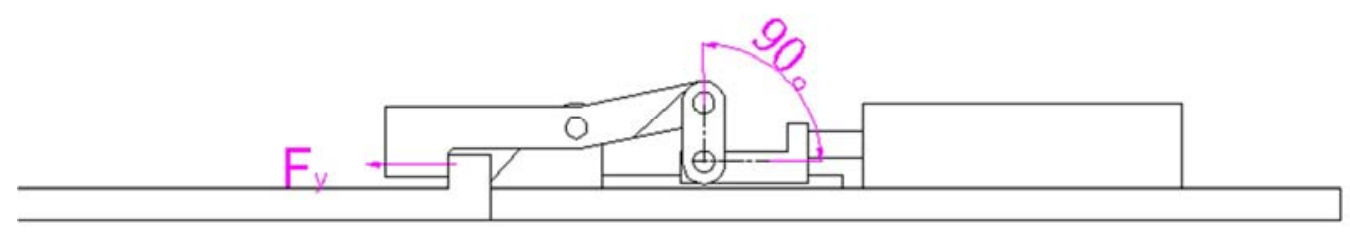

图8 舱段连接紧固中机构状态。

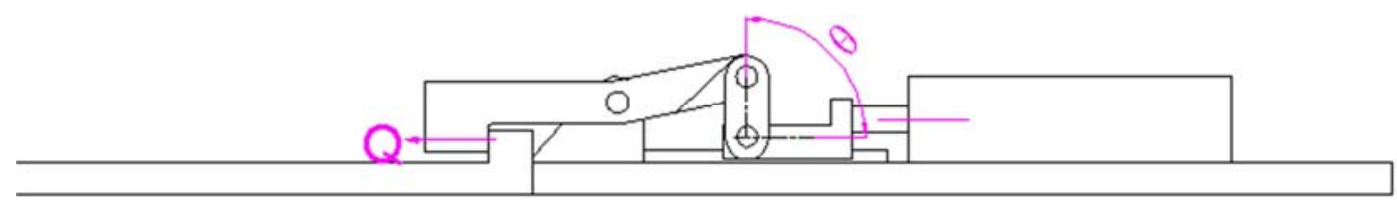

图9 压紧手抓接触压紧面状态。

\section{3. 强度校核}

\section{1. 受力分析}

设均沿舱段圆周方向均布 60 套该快速连接与分离机构, 每套快速连接与分离机构主要尺寸如图 10 所示。
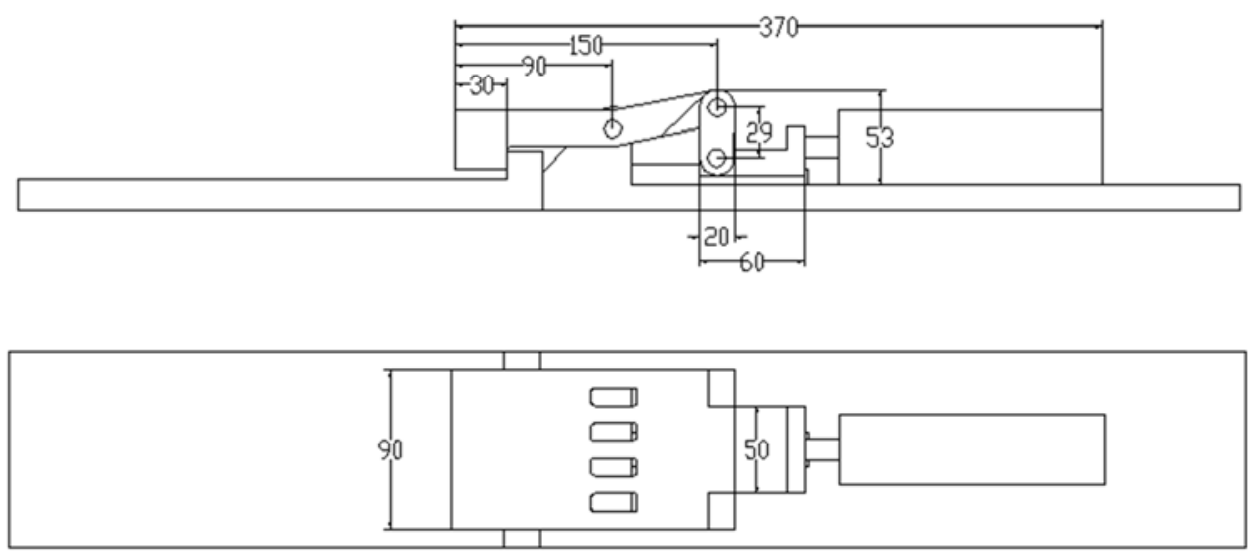

图10 快速连接与分离机构主要尺寸。 

析。

以下对该套快速连接与分离机构的受力情况进行分

舱段承, 最大轴拉力 $\mathrm{Q}$ 拉 $=2.4 \mathrm{X} 106 \mathrm{~N}$, 最大弯矩 $\mathrm{W}=2.8 \times 106$, 最大轴拉力和最大弯矩作用不同时

其中, 由最大轴拉力引发的拉力

$$
\mathrm{F}_{1}=\frac{2.4 \mathrm{X} 10^{6}}{60}=4 \times 10^{4} \mathrm{~N}
$$

离舱段截面中性轴最远的, 由弯矩引发的应力方向一 致处的那套快速连接与分离机构受力最大, 因此由最大弯 矩引发的拉力

由最大弯矩引发的拉力

$$
\begin{aligned}
& \mathrm{F}_{2} \\
& =\frac{2.8 \times 10^{6} / 1.5}{4 \mathrm{X}\left(\left(\sin 6^{\circ}\right)^{2}+\left(\sin 12^{\circ}\right)^{2}+\left(\sin 18^{\circ}\right)^{2}+\cdots+\left(\sin 84^{\circ}\right)^{2}\right)+2} \\
& =62222 \mathrm{~N}
\end{aligned}
$$

因此, 快速连接与分离机构最大受力为:

$$
\mathrm{F}=\max \left(\mathrm{F}_{1}, \mathrm{~F}_{2}\right)=62222 \mathrm{~N}
$$

\section{2. 强度校核}

所有零件均选用高强度不锈钢, $0 \mathrm{Cr} 17 \mathrm{Ni} 4 \mathrm{Cu} 4 \mathrm{Nb}$, 在 $480^{\circ} \mathrm{C}$ 时效处理情况下, 其抗拉强度 $\sigma \mathrm{b} \geq 1310 \mathrm{MPa}$, 条件 屈服强度 $\sigma 0.2 \geq 1180 \mathrm{MPa}$ 。

\subsection{1. 压紧手爪}

压紧手爪的受力情况如图11所示。

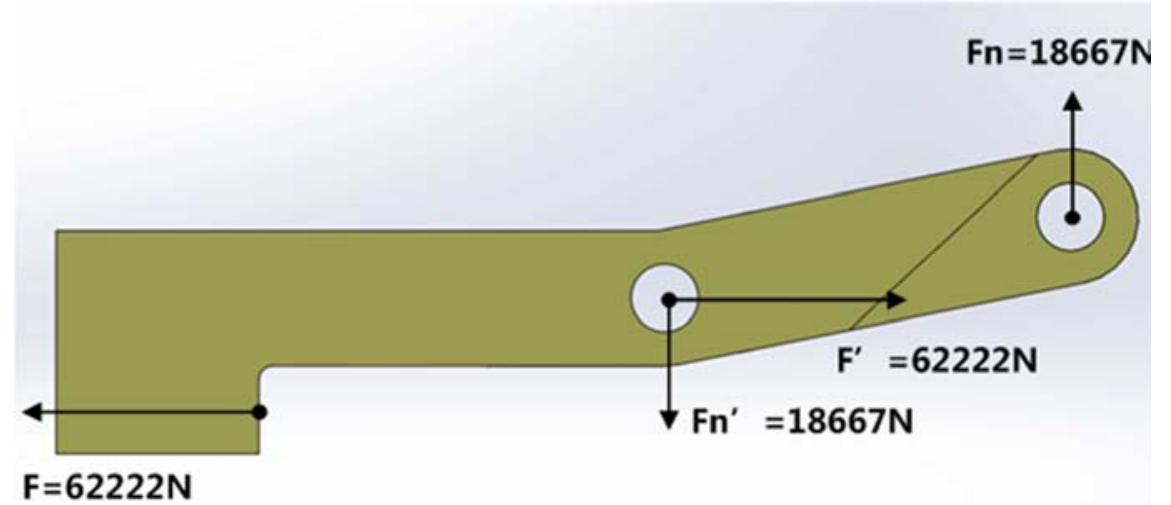

图11 压紧手爪受力图。

其应力分布和变形分布分别如图12所示, 压紧手爪的 最大应力为 $367 \mathrm{MPa}$ ，最大位移为 $0.56 \mathrm{~mm}$ 。
因此压紧手抓结构静承载能力满足要求, 变形较小, 变形量满足使用要求。






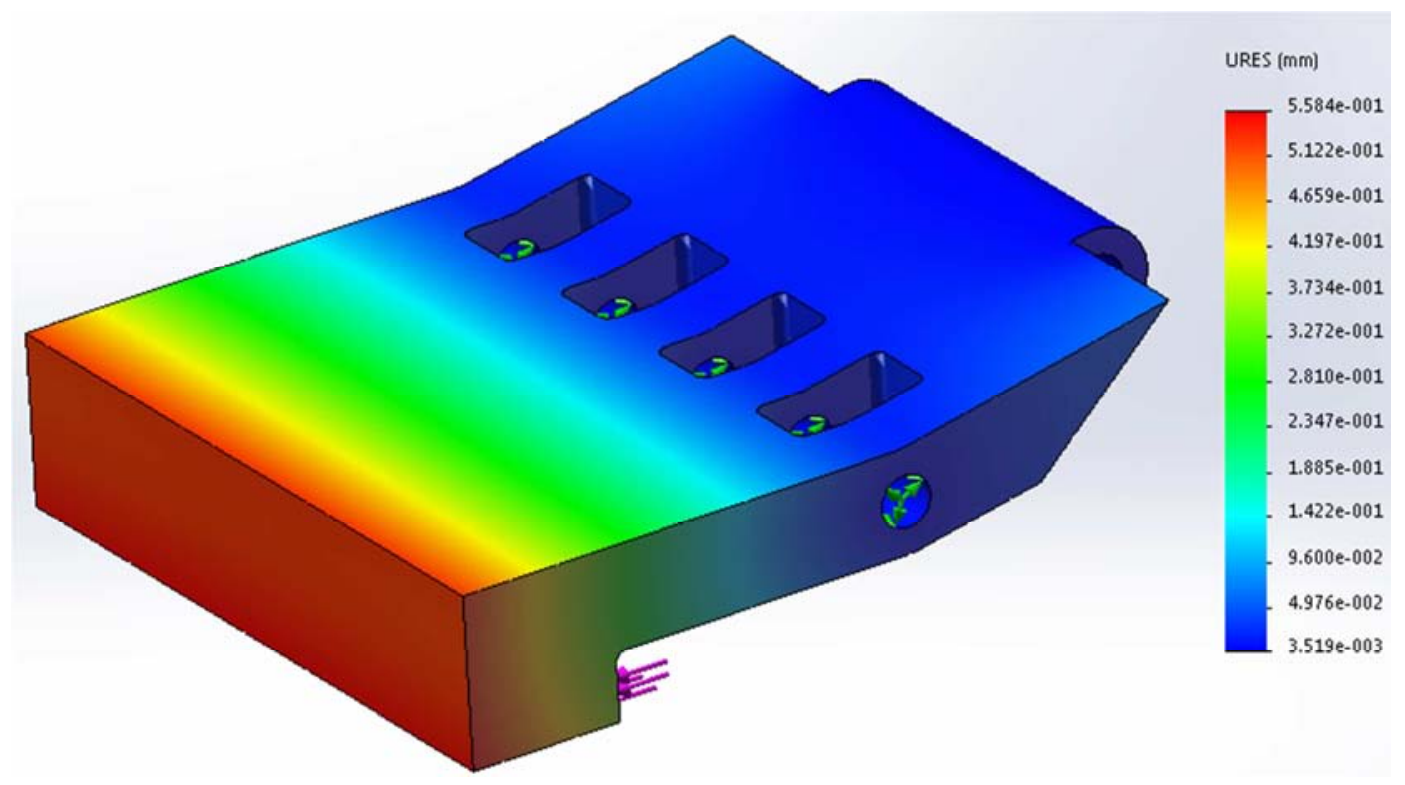

图12 压紧手爪应力分布图和变形分布图。

\subsection{2. 固定支架}

固定支架的受力情况如图13所示。

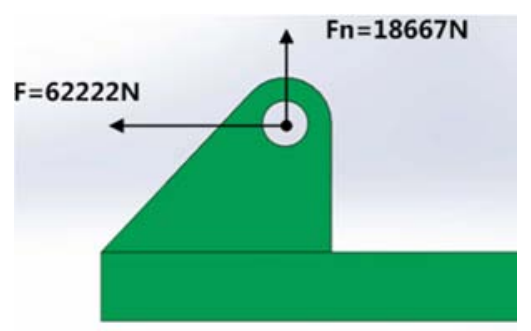

图13 固定支架受力图。

其应力分布和变形分布分别如图 14 所示。由图中可知, 固定支架的最大应力为 $730 \mathrm{MPa}$, 最大变形为 $0.13 \mathrm{~mm}$ 。因 此固定支架结构静承载能力满足要求, 变形较小, 变形量满足使用要求。

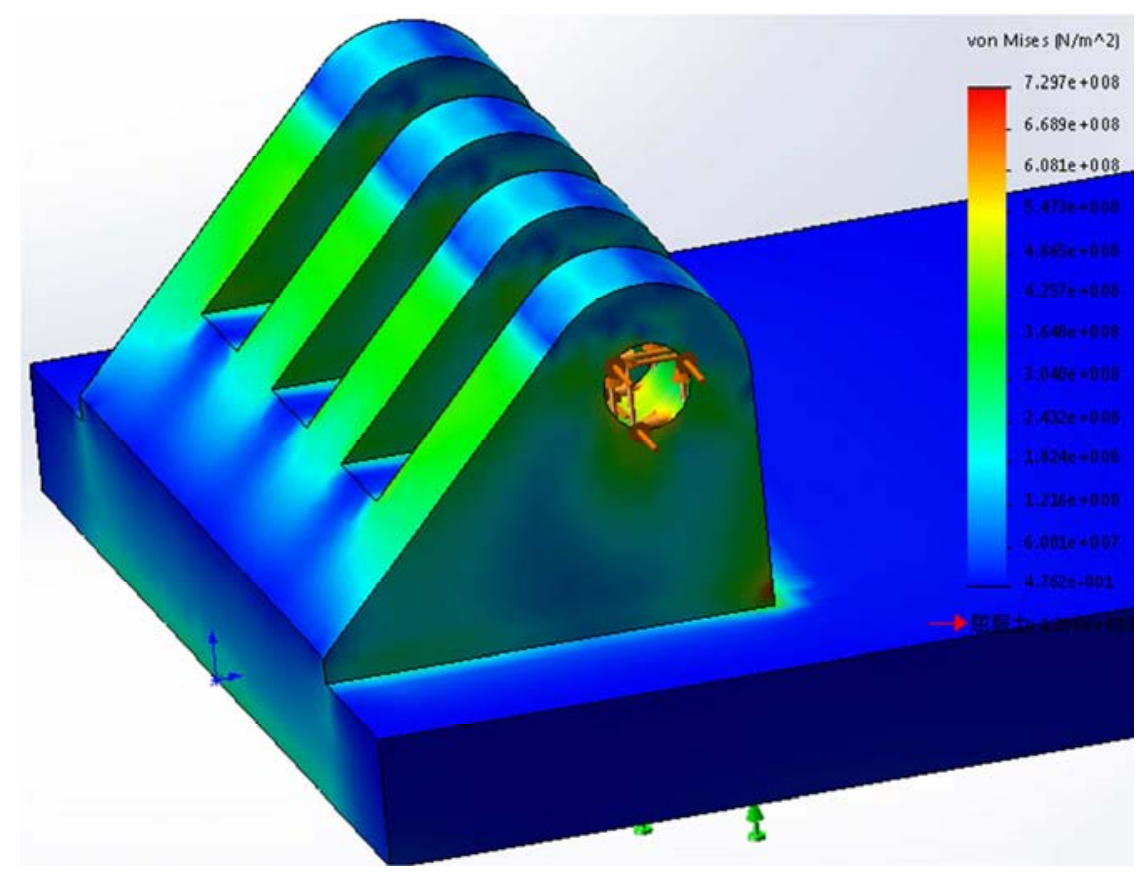




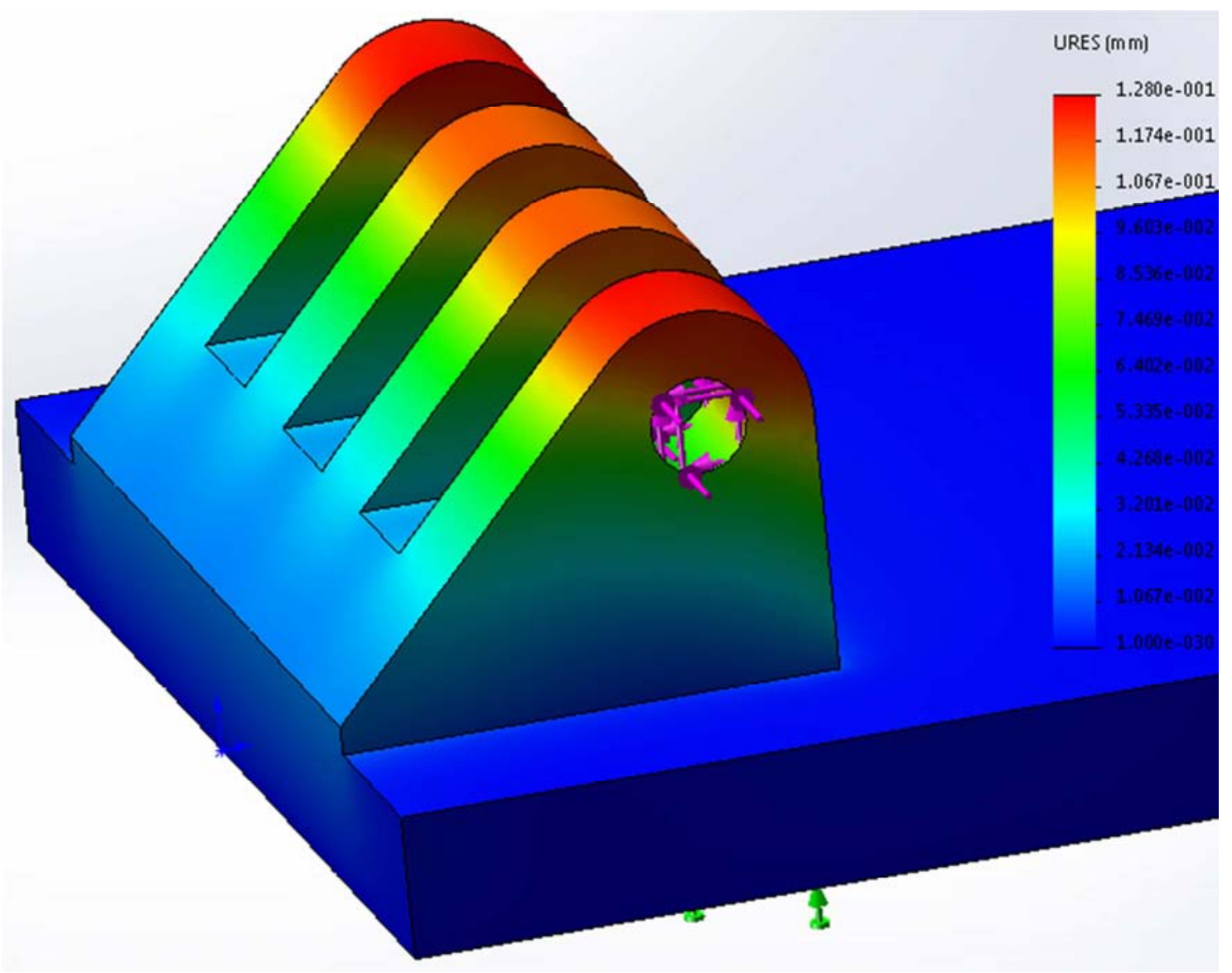

图14 固定支架应力分布图和变形分布图。

\subsection{3. 连杆}

连杆的受力情况如图15所示。

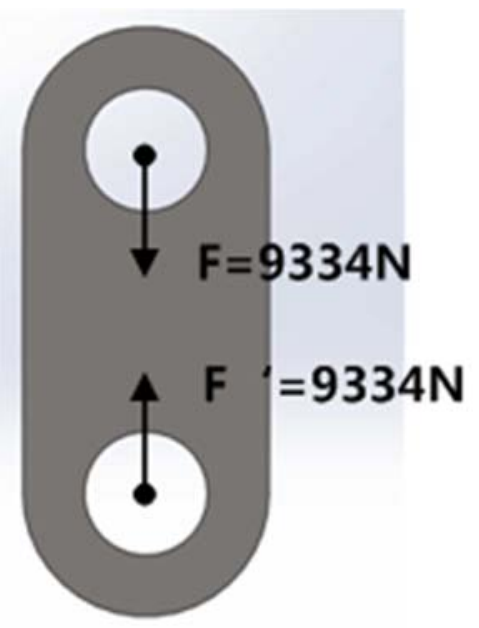

图15 连杆受力图。

其应力分布和变形分布分别如图 16 所示。由图中可知, 连杆的最大应力为 $46 \mathrm{MPa}$, 最大变形为 $0.004 \mathrm{~mm}$, 因此连 杆结构静承载能力满足要求, 变形很小, 变形量满足使用要求。 

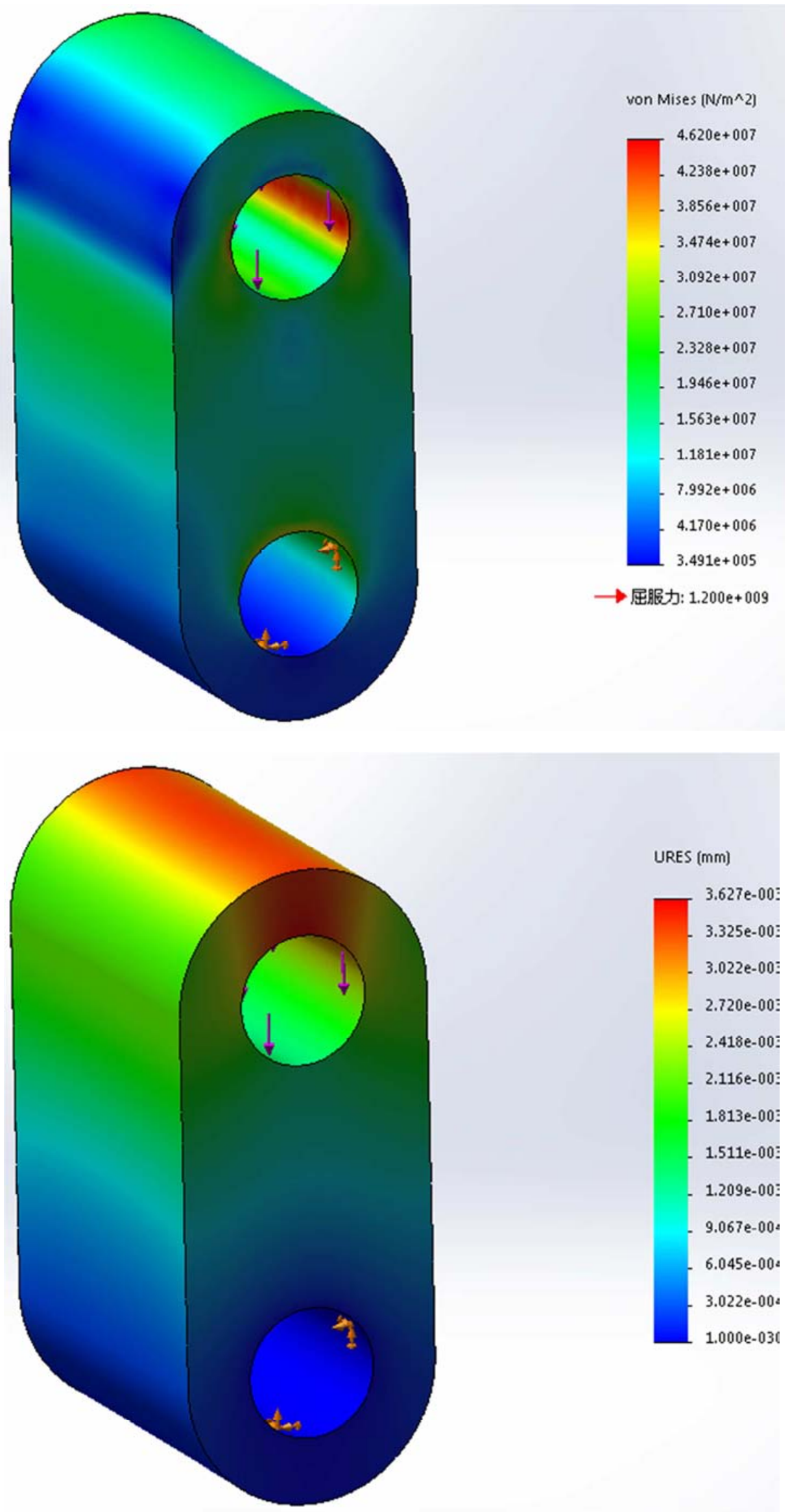

图16 连杆应力分布图和变形图。 


\subsection{4. 滑块}

滑块的受力情况如图17所示。

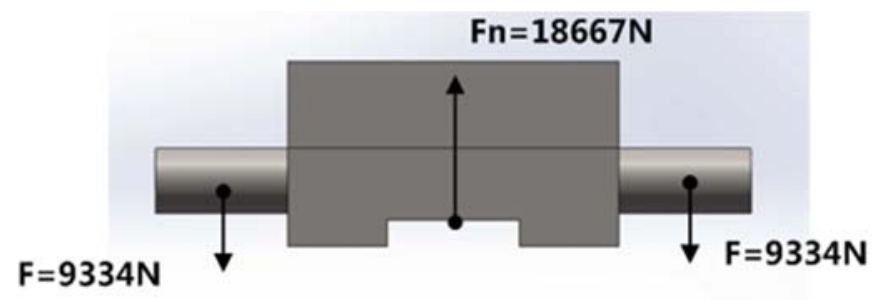

图17 滑块受力图。

其应力分布和位移分布分别如图 18 所示。由图中可知, 滑块的最大应力为 $1079 \mathrm{MPa}$, 最大变形为 $0.3 \mathrm{~mm}$ 。因此滑 块结构静承载能力满足要求, 变形较小, 变形量满足使用要求。
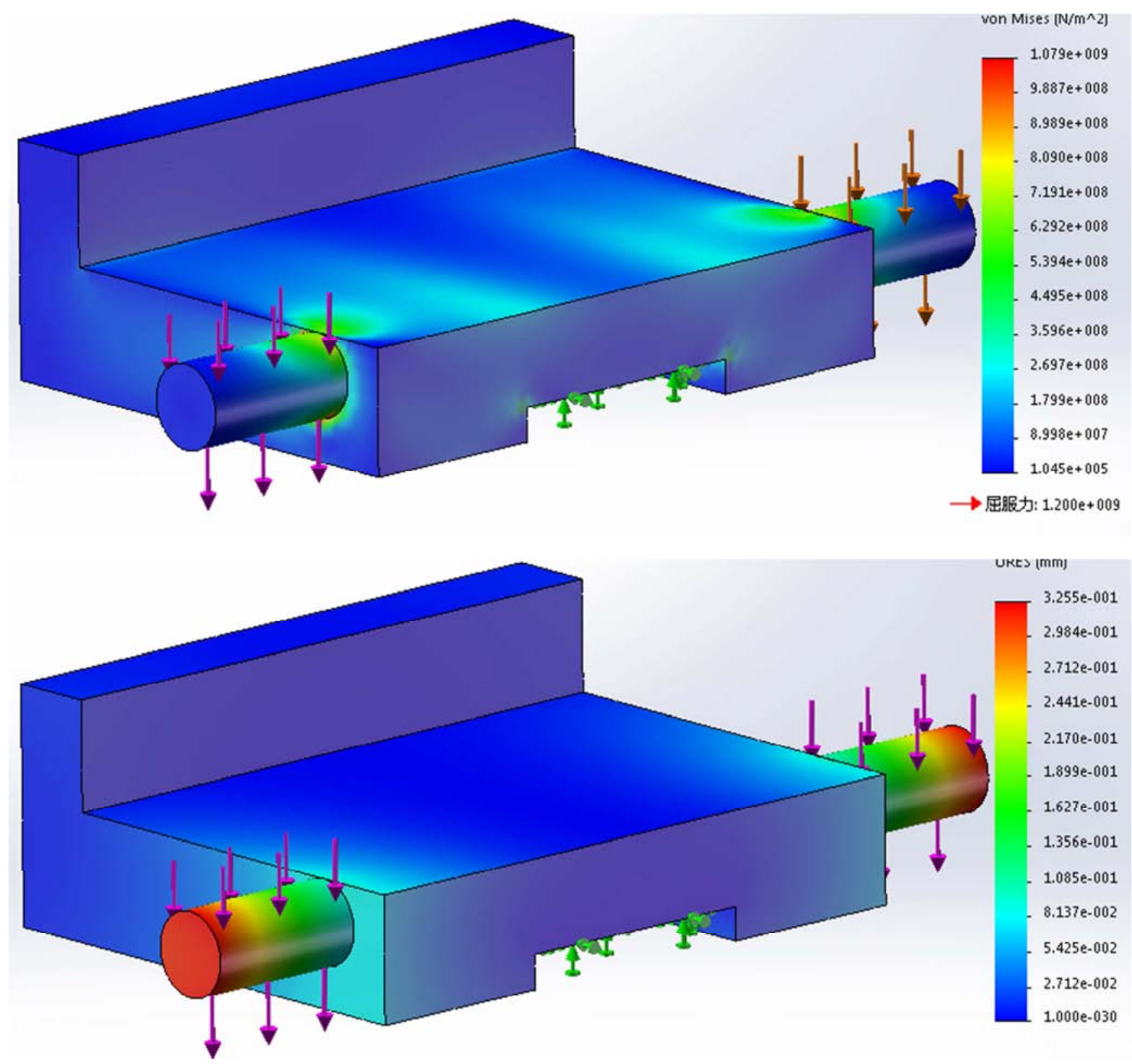

图18 滑块应力分布图和变形分布图。 
综上所述, 本舱段自动快速对接与分离装置的结构静 承载能力以及变形量满足要求。

\section{4. 结论}

为满足级间段模块化快速组合需求, 通过结构设计和 力学分析, 设计出一种新型的舱段自动快速对接与分离装 置。本装置采用直线电机驱动, 布局在狭小的径向空间内, 同时具有舱段对接与分离的功能, 可实现舱段自动快速对 接和多次低冲击分离, 有巨大的工程应用前景。为了达到 使用要求, 后期还需对本装置的整体结构进一步优化, 降 低装置质量, 提高承载能力, 进行性能试验等。

\section{参考文献}

[1] 王振威.基于两瓣式分瓣螺母的圥余电机驱动型星箭分离 装置的设计.哈尔滨工业大学硕士论文.2018

[2] 刘会英. $冗$ 余电机驱动型分离装置的设计与试验. 机械设计 与研究.2016年06期

[3] 王珊; 王栋.导弹舱段间复杂连接件弯曲应力分析. 战术导弹 技术. 2017 年 05 期

[4] 王晓慧; 牀思思.基于混合变量的导弹舱段径向连接结构综 合优化设计.固体火箭技术.2015年06期

[5] 邱凯; 曹鲁光等.导弹关键舱段螺栓联接的有限元分析及预 紧力研究.机械设计与制造.2015年02期
［6］易龙; 彭云等.基于APDL的圆截面舱段楔块连接结构全接 触分析技术研究. 南昌航空大学学报(自然科学版).2017年 01期

[7] 任凯; 洪春风.纯水冷却设备中的电动连杆机构设计.山东化 工.2018年01期

[8] 叶耀坤; 刘天雄等.航天火工连接分离机构设计技术研究. 载人航天.2016年 01 期

[9] 杨国军; 杨桂林等,面向圥余驱动的自适应柔性连接机构优 化设计.制造业自动化.2015年13期

[10] 唐杰; 康士朋等.微纳卫星标准接口连接分离机构技术研究. 国际太空.2016年08期

[11] 吴少杰.某增压机型曲柄连杆机构优化设计. 华南理工大 学.2017年 02期

\section{作者简介}

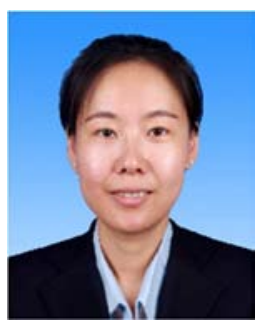

周蓓 (1980-) , 女, 高级工程师, 主 要研究方向为: 航天器武器总体结构设 计 